\title{
VERÄNDERUNGEN IN DER FREQUENZ UND FUNKTION DENDRITISCHER ZELLSUBTYPEN IN SCCHN, DEN ASSOZIIERTEN LYMPHKNOTEN UND PERIPHEREM BLUT
}

\author{
Hendrik Graefe ${ }^{1}$, Evelyn Hartmann², Ralph Pries ${ }^{1}$, Barbara Wollenberg ${ }^{1}$
}

\author{
${ }^{1}$ Universitätsklinikum Schleswig-Holstein, Campus Lübeck. Klinik und Poliklinik für Hals-, Nasen- und Ohrenheilkunde \\ Direktorin: Frau Prof. Dr. B. Wollenberg \\ ${ }^{2}$ Klinik und Poliklinik für Hals-Nasen-Ohrenheilkunde der Ludwig-Maximilians-Universität München \\ Direktor: Herr Prof. Dr. A. Berghaus
}

\section{EINLEITUNG}

Plattenepithelkarzinome des Kopf-Hals-Bereiches ( $\mathrm{SCCHN}$ ) werden von dendritischen Zellen (DC) infiltriert und können diese über bislang nur teilweise geklärte Mechanismen massiv in ihrem Phänotyp und ihrer Funktion verändern Insbesondere ist hierbei die Expression kostimulatorischer Moleküle auf deren Oberfläche für die Interaktion mit T-Zellen und deren Beeinflussung durch CPG-Motive von Bedeutung (Abb. 1). Eine Charakterisierung und Quantifizierung der dendritischen Zellen von SCCHN, der drainierenden Lymphknoten und des peripheren Blutes lässt Rückschlüsse auf Interaktionen des Tumors zu und bietet Ansatzpunkte für zukünftige Immuntherapien.

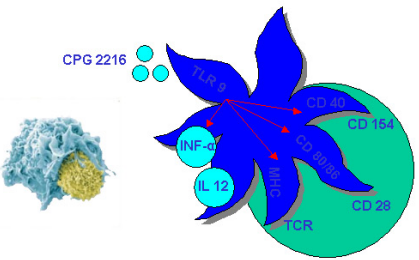

Abbildung 1: Interaktion einer plasmacytoiden dendritischen Zelle mit einer T-Zelle

(elektronenmikroskopische Abbildung von science online)

\section{MATERIAL UND METHODEN}

Intraoperativ wurden Tumor-, Lymphknoten- und Blutproben von Patienten mit SCCHN gewonnen. Als Kontrolle dienten Blutproben von gesunden Probanden. Es wurden dabei nur Proben von Patienten mit einem Erstkarzinom im HNO Bereich gesammelt, um Beeinflussungen durch Radiatio oder Chemotherapie auszuschließen. Unter Einsatz von mechanischer Zerkleinerung sowie enzymatischer Verdauung wurden die dendritischen Zell-Subtypen für die weitere durchflusszytometrische Analyse zur 6Farbenanalyse mittels FACS-Canto aufgeschlossen (Abb. 2). Neben der Klassifizierung der DC mit CD123 (myeloide DC $\mathrm{MDC}$ ), lineage-Cocktail (CD3, CD14, CD16 CD19, CD20, und CD56), CD11c (plasmacytoide DC, PDC) und anti-HLA-DR wurden die Zellen auch auf die Expression der Oberflächenmarker CD86, CD80 und CD40 untersucht (Abb. 3). In Kurzzeitkulturen wurden Stimulationsversuche mit dem Oligonukleotid CPG 2216 in vitro unternommen.

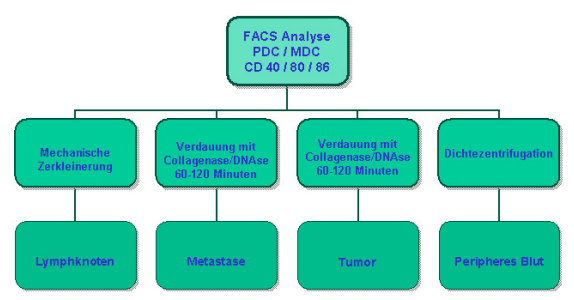

Abbildung 2: Übersicht über die gesammelten Proben und deren Aufschließung zur FACS-Analyse

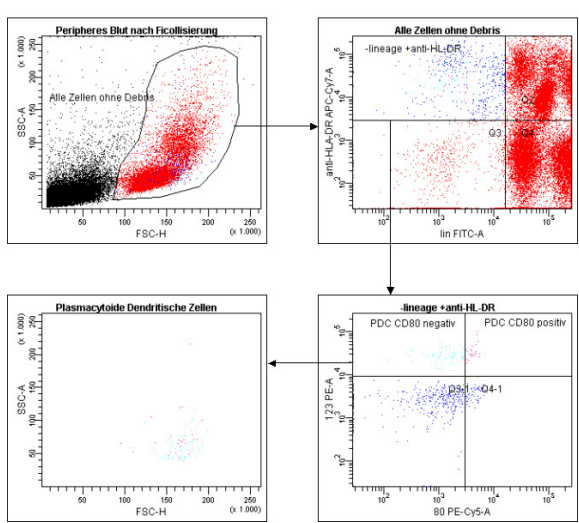

Abbildung 3: Gatingstrategie zur Detektion dendritischer Zellen und zur Quantifizierung der Oberflächenmarke

\section{ERGEBNISSE}

Es wurden Proben von 6 unterschiedlichen Patienten untersucht (Tab. 1). In den Tumorproben und den Lymphknoten-Metastasen fand sich eine signifikant reduzierte Anzahl von PDC und MDC im Vergleich zu den Lymphknotenund Blutproben (Abb. 4).

Durchflusszytometrisch wurde zusätzlich die Ausprägungen der kostimulatorischen Moleküle (CD40/80/86) auf den DC analysiert, dabei zeigte sich eine Reduktion auf den DC in den Tumoren und Metastasen (Abb. 5).

Nach einer 24-stündigen Inkubation der aus Blut, Lymphknoten und Tumor gewonnenen PDCEinzelzellsuspensionen mit CPG 2216 oder Mediumzusatz als Negativkontrolle zeigte sich bei allen Geweben eine deutliche Zunahme der Expression von $\mathrm{CD} 40,80$ und 86 im Vergleich mit den Negativkontrollen (Abb. 6).

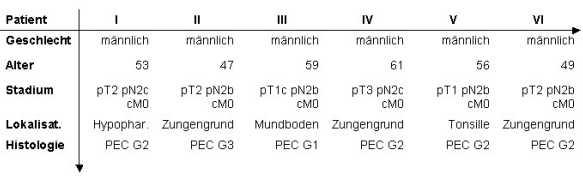

Tabelle 1: Übersicht über das Patientenkollektiv

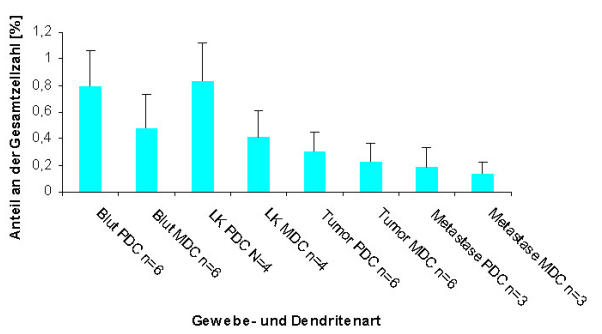

Abbildung 4: Verteilung der DC in den untersuchten Geweben mit Darstellung des Mittelwertes und der Standardabweichung

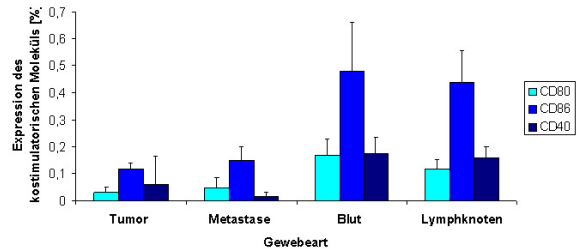

Abbildung 5: Expression von kostimulatorischen Molekülen (Mittelwert und Standardabweichung)

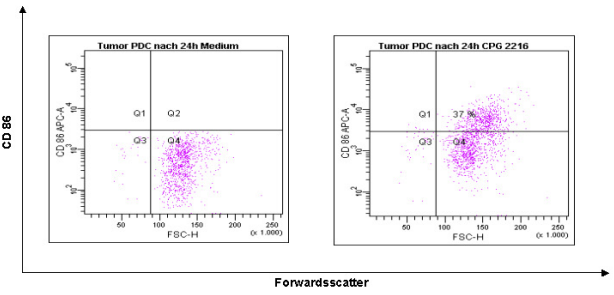

Abbildung 6: Stimulation von PDC im Tumor durch CPG 2216. Andere Daten (CD 80 / 40, Lymphknoten, Blut) werden nicht gezeigt

\section{DISKUSSION}

Bei Patienten mit SCCHN zeigt sich ein deutlich reduziertes Vorkommen von DCs im Tumor und seinen assoziierten Lymphknoten gegenüber dem peripheren Blut. Die Expression der kostimulatorischen Moleküle CD40, CD80 und CD86 war auf PDC im Tumor und den assoziierten Lymphknoten im Vergleich zum peripheren Blut stark reduziert. Eine suffiziente Präsentation von Tumorantigenen an T-Zellen ist aufgrund der eingeschränkten Präsentation der kostimulatorischen Moleküle nicht möglich. Die TZell-Aktivierung und damit eine effiziente T-ZellAntwort ist somit beeinträchtigt.

Durch in vitro Stimulation mit CPG konnte die Expression von CD40/80 und 86 aber wieder erheblich gesteigert werden (Abb. 6).

Über bislang ungeklärte Mechanismen können SCCHN die Quantität und Funktionalität tumorinfiltrierender DC verändern und so massiv auf die Immunabwehr Einfluss nehmen.

\section{AUSBLICK}

Die weitere Analyse phänotypischer und funktioneller Marker, sowie die Umkehr derartiger "Immune-escape" Phänomene bestimmen die zukünftigen immuntherapeutischen Ansätze, wie $z$.B. die intratumorale Stimulation der DCs mit CPG-Oligonukleotiden oder einzelsträngiger RNA.

\section{KONTAKT}

Dr. med. Hendrik Graefe Klinik für Hals- Nasen- und Ohrenheilkunde des UKSH -Campus LübeckRatzeburger Allee 160, 23538 Lübeck Tel.: 0451500 2041; Fax:: 04515002049 Hendrik.Graefe@hno.uni-luebeck.de

Literatur beim Autor

Unterstützt durch die Mildred-Scheel-Stiftung (Deutsche Krebshilfe) 10-2074-Wo2 